\title{
Cardinality of the Continuum of Closed Superclasses of Some Minimal Classes in the Partially Ordered Set $\mathcal{L}_{2}^{3}$
}

\author{
A. V. Makarov ${ }^{a, *}$ and V. V. Makarov ${ }^{b, * *}$ \\ ${ }^{a}$ Moscow State University, Faculty of Mechanics and Mathematics, \\ Leninskie Gory, Moscow, 119991 Russia, ${ }^{*}$ e-mail:vvmavmmakarov@yandex.ru \\ ${ }^{b}$ MIREA - Russian Technological University, \\ Institute for Integrated Security and Special Instrumentation, \\ Stromynka st.20, Moscow, 107996 Russia, ${ }^{* *}$ e-mail:vvmavmmakarov@yandex.ru
}

Received October 24, 2018

\begin{abstract}
It is proved that the set of closed classes containing some minimal classes in the partially ordered set $\mathcal{L}_{2}^{3}$ of closed classes in the three-valued logic that can be homomorphically mapped onto the two-valued logic has the cardinality of continuum.
\end{abstract}

DOI: $10.3103 /$ S0027132219040089

All necessary definitions can be found in the introduction to [1]. It was also proved in [1] that the partially ordered set (further, p.o.s.) $\mathcal{L}_{2}^{3}$ of all closed classes of the three-valued logic $\mathfrak{P}_{3}$ that can be mapped to the two-valued logic $\mathfrak{P}_{2}$ contains only a finite number of minimal elements each of which has the basis composed of one function of two variables. All minimal classes in the p.o.s. $\mathcal{L}_{2}^{3}$ were described in [2]. There were 15 of them.

The countability of the number of closed superclasses of some minimal classes in the partially ordered set $\mathcal{L}_{2}^{3}$ was proved in [3].

In this paper we prove the following

Theorem. The set of closed classes containing any of three minimal classes has the cardinality of continuum. One of these three classes is generated by the following function:

$$
\psi\left(x_{1}, x_{2}\right)=\left(\begin{array}{lll}
1 & 0 & 2 \\
0 & 0 & 2 \\
2 & 2 & 2
\end{array}\right) .
$$

The other two classes are obtained by isomorphic mappings of this class generated by permutations of the cyclic group $\mathbf{A}_{\mathbf{3}}$ of the third order generated by the permutation $\left(\begin{array}{lll}0 & 1 & 2 \\ 1 & 2 & 0\end{array}\right)$.

Note that the matrix $\left(\begin{array}{lll}a_{00} & a_{01} & a_{02} \\ a_{10} & a_{11} & a_{12} \\ a_{20} & a_{21} & a_{22}\end{array}\right)$ defines the function $\varphi(x, y)$ by the relation $\varphi(x, y)=a_{x y}$.

Proof. It was proved in [4] that the set of closed classes of the three-valued logic containing the function of maximum for the partial order $\{(0,2),(1,2)\}$ has the cardinality of continuum. The structure of inclusion of these classes was also described in that paper. It is easy to check that the function $\psi\left(x_{1}, x_{2}\right)$ is obtained by superposition from this function and vice versa.

\section{REFERENCES}

1. A. V. Makarov, "The Homomorphisms of Functional Systems of Multi-Valued Logics," in: Mathematical Problems of Cybernetics, Issue 4, Ed. by S. V. Yablonskii (Nauka, Moscow, 1992), pp. 5-29.

2. A. V. Makarov, "The Description of all Minimal Classes in the Partly Ordered Set $\mathcal{L}_{2}^{3}$ of Closed Classes of the Three-Valued Logic that can be Homomorphically Mapped onto the Two-Valued Logic," Vestn. Mosk. Univ., Matem. Mekhan., No. 1, 65 (2015) [Moscow Univ. Math. Bull. 70 (1), 48 (2015)].

3. A. V. Makarov and V. V. Makarov, "Countability of the Set of Closed Overclasses of Some Minimal Classes in the Partly Ordered Set $\mathcal{L}_{2}^{3}$ of All Closed Classes of Three-Valued Logic that Can be Mapped Homomorphically onto Two-Valued Logic," Vestn. Mosk. Univ., Matem. Mekhan., No. 1, 62 (2017)

4. G. V. Bokov, "Lattice of Closed Classes of Three-Valued Logic Containing The Function of Maximum for a Nonlinear Partial Order," in: Proc. XII Int. Workshop "Discrete Mathematics and its Applications" named after Acad. O. B. Lupanov (Moscow State Univ., Moscow, 2016), pp. 187-190.

Translated by V. Valedinskii 